\title{
Dermal sinus tracts of the spine
}

\author{
Scott Elton, M.D., AND W. Jerry OAKes, M.D. \\ Departments of Neurosurgery and Pediatric Neurosurgery, Children's Hospital, Birmingham, \\ Alabama
}

\begin{abstract}
Dermal sinus tracts in the spine range from asymptomatic pits to tracts with significant disease. These tracts may be associated with lesions that tether the spinal cord and can either become infected or produce neurological deficits. Over time the treatment of these lesions has varied little: complete resection and intradural exploration are the standard surgical interventions. The authors review their experience with 23 dermal sinus tracts treated in the last 19 years by the senior author. The clinical findings, radiographic appearance, treatment, and pathological findings of these lesions will be discussed. The authors will also provide a summary of the literature covering these lesions.
\end{abstract}

KEY WoRDS - dermal sinus tract - spinal dysraphism • dermoid cyst

Dermal sinus tracts are remnants of incomplete neural tube closure. Embryologically, they result from a failure of the surface ectoderm and dermal elements to separate from the neuroectoderm. This process likely occurs between the 3rd and 8th weeks of gestation. Dermal sinus tracts may be associated with spinal fluid drainage, intradural dermoid or epidermoid cysts, and spinal cord tethering. Their treatment in the past has consisted of either superficial resection or intradural surgical exploration. As these lesions often transgress the dura mater and are associated with significant intradural disease, the current literature would support intradural exploration. To further understand dermal sinus tracts, we have reviewed our 19year experience with the treatment of patients harboring these lesions.

\section{CLINICAL MATERIAL AND METHODS}

The authors conducted a retrospective chart review of all children seen, evaluated, and treated for spinal dermal sinus tracts between 1981 and 2000 by the senior author (W.J.O.).

\section{RESULTS}

We found a total of 23 patients treated for dermal sinus tracts of the spine. Thirteen were male, and 10 were female. The average age was 6.6 years (range 5 days -38 years). Six (26\%) of 23 patients harbored intradural der-

Abbreviation used in this paper: $\mathrm{MR}=$ magnetic resonance. moid cysts: three were intramedullary, and three were intradural but not involving the spinal cord. Nine (39\%) of 23 presented with other findings of occult spinal dysraphism. There were two children with lipomyelomeningoceles and three with diastematomyelias. In eight of 23 patients, the findings were consistent with a tethered spinal cord. All were surgically treated. There was no incidence of mortality in this series. There were no cases of surgery-related neurological worsening in those children who presented with neurological deficit. No patients suffered a neurological insult as a result of intervention. No other deficits were incurred.

\section{ILLUSTRATIVE CASES}

\section{Case 1}

This female patient was a neonate in whom excision of a cervical dermal sinus tract had been performed. The neurosurgeon who resected the superficial lesion did not find a stalk penetrating the cervical fascia, and no further exploration was performed. The patient presented again at 5 years of age; she could not use her right arm and this was secondary to disabling sensory loss. Imaging studies revealed a tethered cervical spinal cord (Fig. 1). Despite the surgical untethering of the band fixating the cord dorsally, the patient's sensory loss, as well as the disuse of her arm, continued. The results obtained in this case illustrate the need for careful intradural exploration and very precise dissection of the subcutaneous sinus tract to avoid such outcomes. 


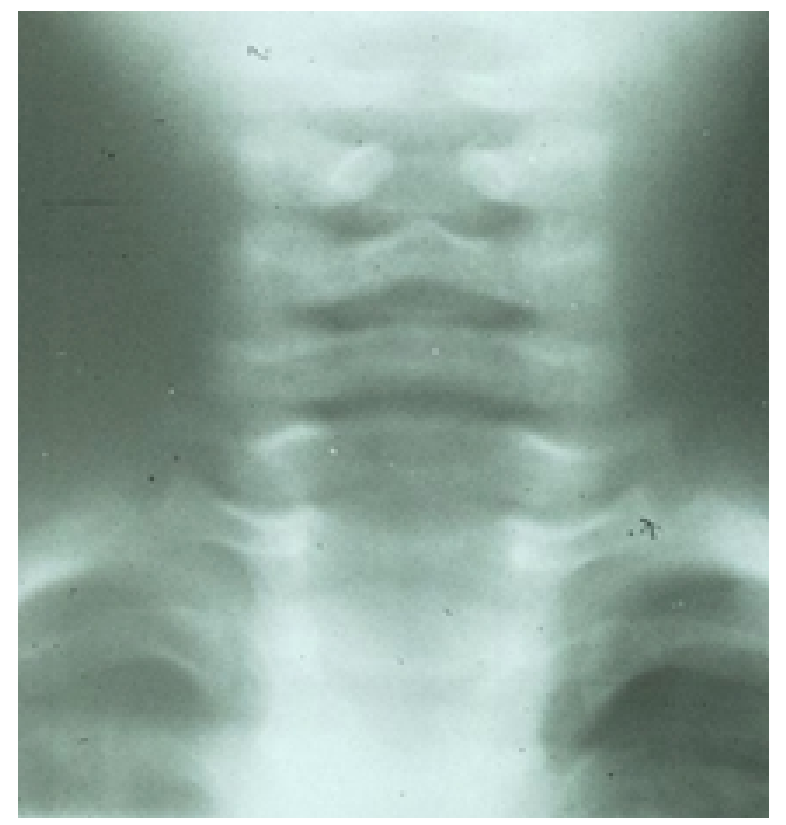

Fig. 1. Case 1. Radiograph demonstrating a bifid cervical spine in a patient who underwent superficial excision of dermoid cyst only. With this type of bone defect intradural exploration is mandatory.

\section{Case 2}

This female neonate presented to a neurology service with the acute onset of meningitis and paraplegia. After repeated examination, she was found to have a small dermal sinus tract (Fig. 2). Imaging studies revealed an intramedullary dermoid cyst that became infected and acted as a compressive mass abscess resulting in the paraplegia. Although not pathognomonic for an infected dermoid, the acute presentation of paraplegia and meningitis is highly suggestive, particularly in the presence of the cutaneous stigmata, of dermal sinus tract.

\section{Case 3}

This male patient presented at 1 year of age with men-

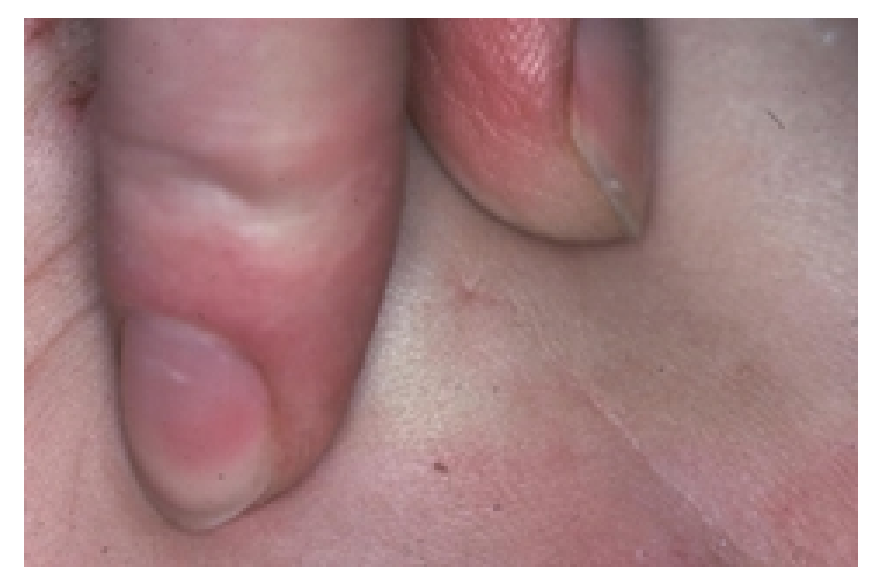

Fig. 2. Case 2. Photograph showing minuscule dermal sinus tract in patient presenting with paraplegia and infected dermoid cyst.

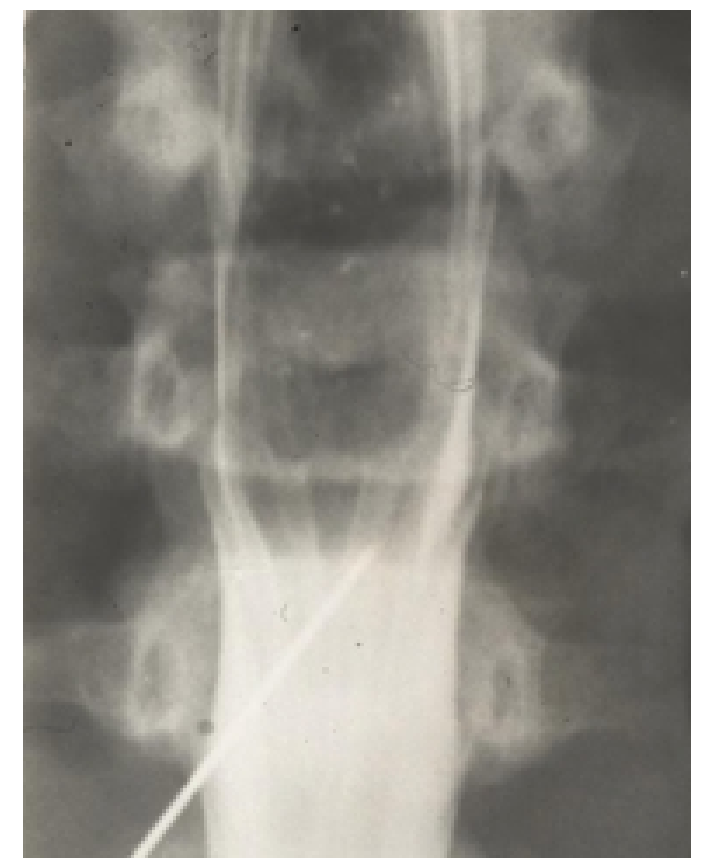

Fig. 3. Case 3. Myelography study demonstrating an intradural dermoid cyst 11 years after the patient underwent excision of a superficial dermal sinus tract.

ingitis. He was found to have a dermal sinus tract. The patient was treated with antibiotic therapy. When he was clinically well 5 days after the initiation of therapy with a normal white blood cell count, resection of his dermal sinus tract was performed. It is important in the presence of active meningitis to wait to resect the lesion until the patient's clinical condition has stabilized. Meningitis predisposes to a brisk inflammatory reaction, producing dense arachnoidal scarring, and making operations months and years after the infection significantly more difficult. In addition, waiting to operate exposes the patient to the risk of a recurrent infection.

\section{Case 4}

This male patient underwent a superficial excision of his dermal sinus tract as an infant. Lost to medical follow up, he presented again at age 11 years with a neurogenic bladder. Imaging studies revealed an intradural dermoid (Fig. 3), and he underwent resection of this lesion. This case underscores the need for evaluation of the intradural compartment in patients with dermal sinus tracts, as intracanal dermoid and epidermoid cysts are not unusual in this population.

\section{Case 5}

This patient presented with meningitis as a neonate. Workup revealed a lumbar dermal sinus tract, although none was visualized on imaging studies. At surgery, a small tract was discovered. This tract ascended to the conus medullaris in the subarachnoid space (Fig. 4). These lesions may have tracts that are located below the resolution, or out of the plane of MR imaging. When surgically treating these lesions, it is important to recognize 


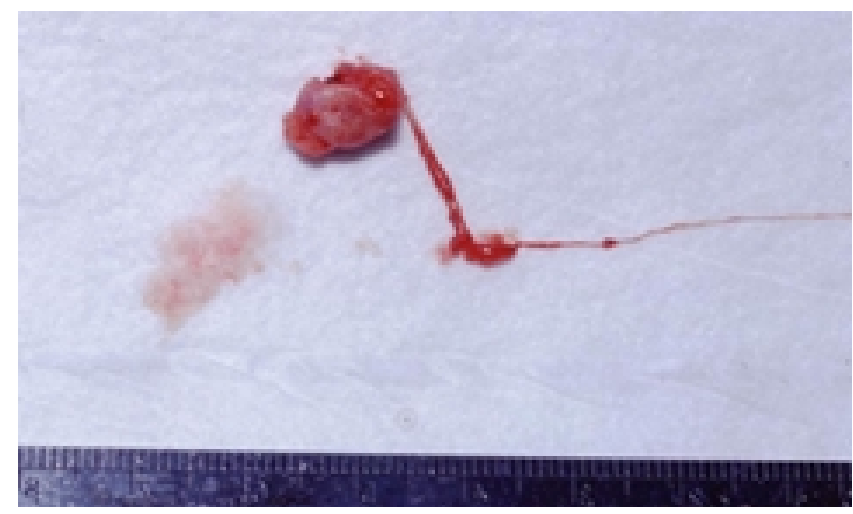

Fig. 4. Photograph showing a sacral dermoid cyst with small stalk that became attached to the conus medullaris.

that a very small tract may penetrate the fascia, and care should be taken to explore the extent of the tract.

\section{Case 6}

This patient had undergone two prior resections of a sacral dermoid cyst associated with a dermal sinus tract. He presented to us with disabling lower-extremity pain. There were no symptoms of neurogenic bladder. Evaluation revealed craniospinal dissemination of the dermoid cyst (Fig. 5). He has remained neurologically intact, despite the dissemination, but is incapacitated by pain caused by dense arachnoiditis, which is associated with contamination of the subarachnoid space by the contents of the dermoid cyst.

\section{DISCUSSION}

Spinal dermal sinus tracts consist of an epithelial-lined tract extending from the skin surface to the spinal fascia, dura mater, or spinal cord. These lesions may be found anywhere along the midline of the neural axis. They result from abnormal ectodermal adhesion of the neural tube to the dermis. Dermal tissue may then go on to develop within the trapped ectodermal tract. Although the incidence of sacrococcygeal pits may be $4 \%$ within the neonatal population, the incidence of true congenital dermal sinus tracts is not known, though they are very uncommon. ${ }^{10}$ It is likely that earlier studies have mixed coccygeal pits with dermal sinus tracts. ${ }^{3}$ These two entities, however, are distinct.

The cutaneous opening of a dermal sinus tract differs from that of a coccygeal pit. ${ }^{13}$ Dermal sinus tracts are found above the natal cleft and are usually directed superiorly. By comparison, coccygeal pits are found within the natal cleft with a tract extending either straight down or inferiorly. ${ }^{10,13}$ Coccygeal pits occur over the lower sacrum and coccyx and are anatomically located below the level of the cul-de-sac of the subarachnoid space ${ }^{10}$ (Fig. 6A). Coccygeal pits do not require further imaging evaluation or treatment.

Common cutaneous findings associated with dermal sinus tracts include a pit, flat capillary hemangiomas, hypertrichosis, subcutaneous lipomas, drainage of debris or fluid from the pit, and signs of local infection ${ }^{3,5,10-12}$ (Figs. 6B-D). Infections, both locally as well as meningitis, can

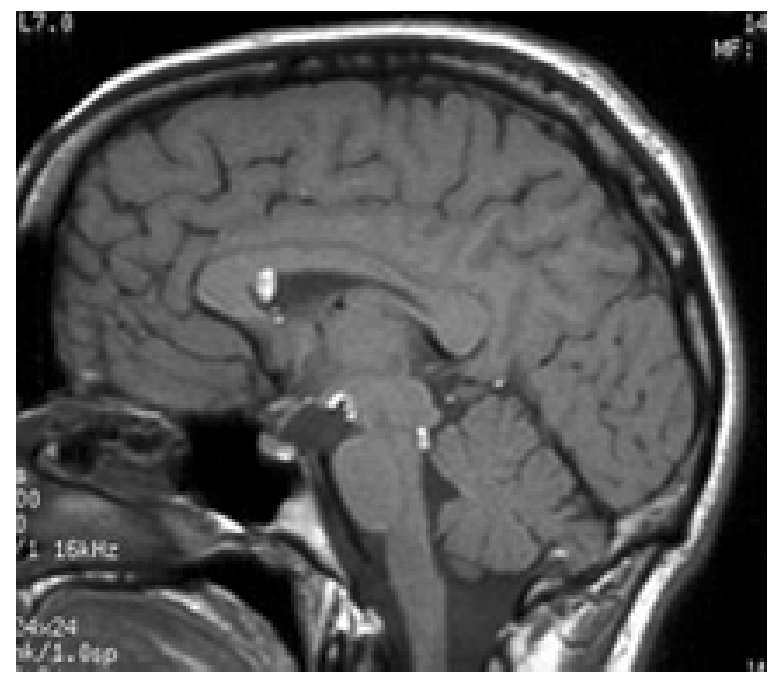

Fig. 5. Sagittal $\mathrm{T}_{1}$-weighted MR image revealing a disseminated intracranial dermoid cyst in patient in whom prior resections of sacral dermoid cyst were incomplete.

be the presenting symptoms of a dermal sinus tract. ${ }^{2,4,9,11}$ The most common organisms cultured include staphylococci, Escherichia coli, and Proteus, although it is possible to acquire polymicrobial infections as well. Chemical meningitis may result from a spontaneous leakage of dermoid cyst contents into the spinal fluid. Either chemical or

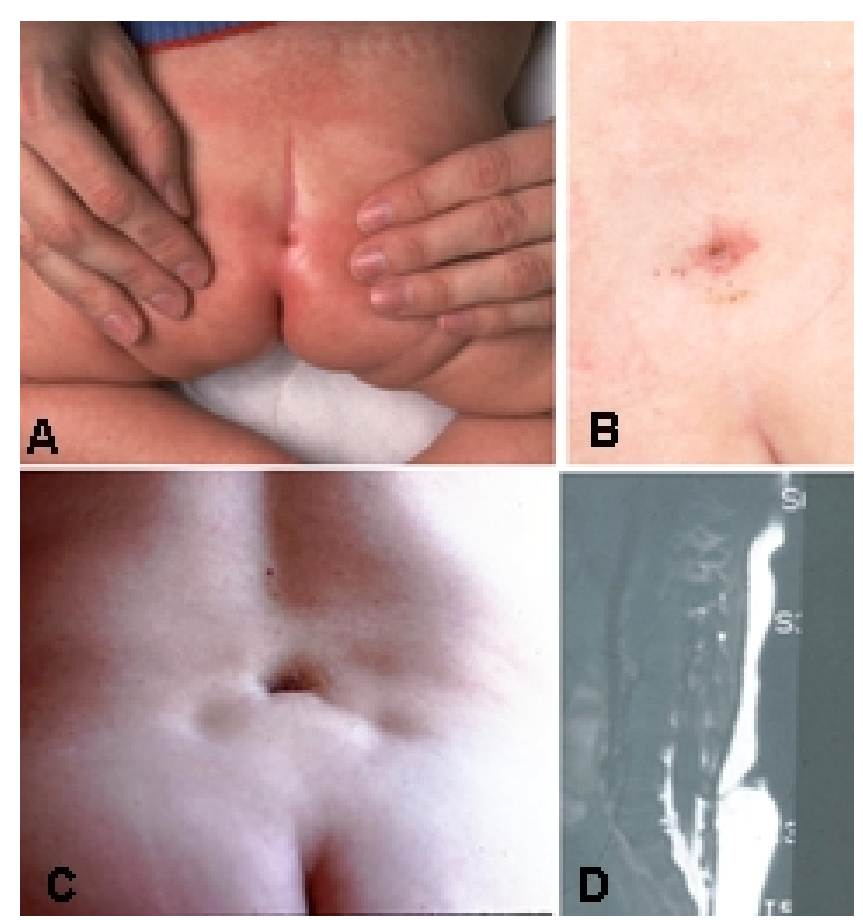

Fig. 6. A: Photograph showing a sacrococcygeal pit within gluteal crease. B: Photograph showing a dermal sinus tract with cutaneous hemangioma superior to gluteal crease. C: Photograph showing a large dermal sinus superior to gluteal crease. D: Sagittal $\mathrm{T}_{\text {}}$-weighted MR image of the lumbar spine demonstrating dermal sinus tract in the subcutaneous tissues. 
infectious meningitis may predispose to hydrocephalus, although the incidence of this has not yet been examined in patients with congenital dermal sinus tracts. The most common neurological finding is spinal cord compression secondary to intradural dermoid cyst growth, although symptoms of tethered cord may also develop..$^{8,10}$ The cauda equina can be compressed, producing symptoms referable to this region.

Clinical diagnosis is dependent on observation of the aforementioned symptoms and findings. Because these lesions usually transgress the dura, probing the lesions is unwise and not recommended. Magnetic resonance imaging is the neurodiagnostic technique of choice, as the images provide significant information on the neural structures and their relationship, if any, to the dermal sinus tract $^{1,6-8}$ The sinus tract may not be well appreciated on MR imaging if it is small or out of the imaging plane . Magnetic resonance imaging will often reveal intradural dermoid cysts. However, findings on MR imaging may be confusing in the presence of ongoing infection or arachnoiditis, either of which can masquerade as an intradural lesion with clumping of the nerve roots. ${ }^{6,8}$

Treatment of these lesions consists of excision of the dimple and the tract, as well as any intradural connections or masses. Even when MR imaging reveals normal findings dermal sinus tracts located above the gluteal crease should be explored to their termination. General procedure-related morbidity is acknowledged to be very low, with postoperative infection being the most common complication, although the number of cases described in the literature is limited. ${ }^{8,10,12}$ The surgical technique consists of removing the cutaneous lesion in an ellipse, and following the underlying tract to the lumbar fascia. If radiological findings indicate that the lesion penetrates the fascia or if this is observed intraoperatively, the dissection should proceed along the tract until its terminus, frequently the conus medullaris, is reached. The risk of intradural exploration is quite low, and exploration may reveal previously unappreciated pathological findings. This is particularly important because the likelihood that an intradural lesion is present in a patient with a congenital dermal sinus tract is high. If intradural lesions such as a tethered spinal cord or dermoid cyst are present, then the dissection must proceed into the intradural space. These lesions can be scarred extensively or, by their nature, firmly attached to the surrounding neural structures. Good surgical planning is very important when evaluating these lesions. Debulking dermoid cysts from within is the method of choice. The capsule, which may be adherent, can usually be dissected from the surrounding neural elements. Retention of an epithelial surface will result in recurrence of the dermoid cyst and necessitate a repeated operation. An attempt to remove the capsule should be undertaken to prevent further recurrence of lesion. The initial dissection has the highest likelihood of achieving complete resection. Although postoperative scarring and arachnoid scar- ring from meningitis limit the extent of further operations, the risk of neurological injury is not unacceptable.

In conclusion, the diagnosis of a spinal dermal sinus tract should prompt further radiological evaluation and surgical intervention. Surgery is warranted in the presence of a dermal sinus tract in a position that suggests intradural extension. At surgery, the tract should be followed intradurally, and the associated dermoid lesion should be excised. The risks associated with surgical exploration are very low and the benefit high. There is no justification for a conservative approach as such a therapy risks the development of meningitis or progressive spinal cord injury in patients with dermal sinus tracts located above the gluteal crease.

\section{References}

1. Barkovich AJ, Edwards MS, Cogen PH: MR evaluation of spinal dermal sinus tracts in children. AJNR 12:123-129, 1991

2. Benzil DL, Epstein MH, Knuckey NW: Intramedullary epidermoid associated with an intramedullary spinal abscess secondary to a dermal sinus. Neurology 30:118-121, 1992

3. Carrillo R, Carriera LM, Prada JJ, et al: Lateral congenital spinal dermal sinus. A new clinical entity. Childs Nerv Syst 1: 238-240, 1985

4. Chen CY, Lin KL, Wang HS, et al: Dermoid cyst with dermal sinus tract complicated with spinal subdural abscess. Pediatr Neurol 20:157-160, 1999

5. Davis DA, Cohen PR, George RE: Cutaneous stigmata of occult spinal dysraphism. J Am Acad Dermatol 31:892-986, 1994

6. Dev R, Husain M, Gupta A, et al: MR of multiple intraspinal abscesses associated with congenital dermal sinus. AJNR 18: 742-743, 1997

7. Hattori H, Higuchi Y, Tashiro Y: Dorsal dermal sinus and dermoid cysts in occult spinal dysraphism. J Pediatr 134:793, 1999

8. Kanev PM, Park TS: Dermoids and dermal sinus tracts of the spine. Neurosurg Clin North Am 6:349-366, 1995

9. Martinez-Lage JF, Esteban JA, Poza M, et al: Congenital dermal sinus associated with an abscessed intramedullary epidermoid cyst in a child: case report and review of the literature. Childs Nerv Syst 11:301-305, 1995

10. McComb JG: Congenital dermal sinus, in Pang D (ed): Disorders of the Pediatric Spine. New York: Raven Press, 1995, pp 349-360

11. Morandi X, Mercier P, Fournier HD, et al: Dermal sinus and intramedullary spinal cord abscess. Report of two cases and review of the literature. Childs Nerv Syst 15:202-206, 1999

12. Wang KC, Yang HJ, Oh CW, et al: Spinal congenital dermal sinus-experience of 5 cases over a period of 10 years. J Korean Med Sci 8:341-347, 1993

13. Weprin BE, Oakes WJ: Coccygeal pits. Pediatrics 105:E69, 2000

Manuscript received December 1, 2000.

Accepted in final form December 15, 2000.

Address reprint requests to: W. Jerry Oakes, M.D., Suite ACC 400, 1600 7th Avenue South, Children's Hospital, Birmingham, Alabama 35233. 